immediate after initiation of cEEG in $15 \%$, within 1 hour in $50 \%$, and within 24 hours in $80 \%$. Patients with clinical seizures prior to cEEG had NCSz more frequently than those without $(83 \%$ vs $17 \%)$. NCSz were most common in patients with periodic lateralized epileptiform discharges (PLEDS) [73\%], absence of background reactivity on cEEG [65\%], any periodic discharges [65\%], and absence of sleep architecture [50\%]. (Jette N, Claassen J, Emerson RG, Hirsch LJ. Frequency and predictors of nonconvulsive seizures during continuous electroencephalographic monitoring in critically ill children. Arch Neurol Dec 2006;63:1750-1755). (Respond: Nathalie Jette MD MSc, Foothills Medical Centre, Department of Clinical Neurosciences, $1403-29^{\text {th }}$ St NW, Calgary, Alberta, Canada T2R 1R8).

COMMENT. Nonconvulsive seizures are common during cEEG in critically ill children, especially in those with clinical seizures before cEEG initiation. Patients with PLEDS should be monitored for at least 24 hours to exclude NCSz.

\title{
HEART RATE CHANGES OF LIMITED VALUE IN DETECTION OF NEONATAL SEIZURES
}

Heart rate changes were studied during 169 seizures in 14 neonates with severe birth asphyxia at the Erasmus MC-Sophia Children's Hospital, Rotterdam, The Netherlands. Changes were detected during 21 seizures $(12.4 \%)$ in 8 patients; heart rate was increased in 4 , decreased in 1, and both patterns were observed in 3 patients. Diminished or absent beatto-beat variability ("stable baseline HR"), recognized as a poor prognostic sign, was associated with a nonsignificant trend toward increased mortality. Heart rate monitoring is of limited sensitivity for detecting postasphyxial neonatal seizures. (Cherian PJ, Blok JH, Swarte RM, Govaert P, Visser GH. Heart rate changes are insensitive for detecting postasphyxial seizures in neonates. Neurology Dec 2006;67:2221-2223). (Reprints: Dr PJ Cherian, Erasmus MC, Department of Clinical Neurophysiology, University Medical Center, Dr Molewaterplein 40, 3015 GD Rotterdam, The Netherlands).

COMMENT. Heart rate changes during seizures involve connections between the cerebral hemispheres and brain stem autonomic regulatory centers. Unlike adults with ictal heart rate and blood pressure changes, of value in differentiating pseudo- from epileptic seizures (Opherk C et al. Neurology 2002;58:636-638; Laroia N. Neurology 2006;67:2101), the connections that govern autonomic dysregulation are insensitive in the neonate with postasphyxial seizures.

\section{ATTENTION DEFICIT AND COGNITIVE DISORDERS}

\section{EFFECT OF ROLANDIC SPIKES ON ADHD IMPULSIVE BEHAVIOR}

The association of Rolandic spikes with the neuropsychological profile of children with attention deficit hyperactivity disorder (ADHD) was studied in a total of 48 patients at JW Goethe-University, Frankfurt/Main; and Central Institute of Mental Health, Mannheim, Germany. Sixteen children with ADHD and Rolandic spikes (RS) were compared with 16 
ADHD children without epileptiform EEG discharges, and 16 healthy controls. The neuropsychological test battery focussed on attentional processing, cognitive efficiency, response inhibition, visuospatial and auditory-verbal short-term memory and language functioning. ADHD children with RS, compared to ADHD without RS and controls, showed impairments in performance of a Continuous Performance Task, with more commission errors, indicating deficient inhibition of an ongoing response, and errors on the Stroop task, with deficient interference control, both pointing to an increased impulsivity (lack of response inhibition). (Holtmann $\mathrm{M}$, Matei $\mathrm{A}$, Hellmann $\mathrm{U}$ et al. Rolandic spikes increase impulsivity in ADHD - A neuropsychological pilot study. Brain Dev Nov 2006;28:633640). (Respond: e-mail: holtmann@em.uni-frankfurt.de (M. Holtmann)).

COMMENT. This study demonstrates two forms of response inhibition in ADHD children with Rolandic spikes (RS) compared to ADHD children without RS and controls. These are deficient inhibition of an ongoing response, and deficient interference control, both deficits pointing to an increased impulsivity, or lack of response inhibition. These findings confirm previous reports of increased distractibility and impulsivity, and impaired inhibitory control in children with Rolandic spikes. (Yung AWY et al. Ped Neurol 2000;23:391-395).

\section{SPECTRUM OF IDIOPATHIC ROLANDIC EPILEPSY SYNDROMES AND BEHAVIORAL CORRELATES}

The natural history of benign childhood epilepsy with centrotemporal spikes (BCECTS), BCECTS Plus syndromes, Rolandic epilepsy-related syndromes, and idiopathic benign childhood epilepsy with occipital spikes (Panayiotopoulos syndrome) is reviewed by researchers at Maggiore Hospital, Bologna, Italy. These syndromes constitute a group of agedependent idiopathic epilepsy disorders with a characteristic EEG pattern and neuropsychological deficits related to ongoing paroxysmal activity. These syndromes forming a continuum or spectrum have a common underlying mechanism, modulated by the duration, localization, and age of onset of the spike-wave discharge. Some consider the syndromes to represent a type of "epileptic encephalopathy." Continuous spike-waves during sleep syndrome (CSWS) is included as an epileptic encephalopathy, a condition in which the epileptiform abnormalities are believed to contribute to a global regression of cognitive function and behavior. The role of the genetic predisposition to BCECTS and related syndromes requires further elucidation. (Gobbi G, Boni A, Filippini M. The spectrum of idiopathic rolandic epilepsy syndromes and idiopathic occipital epilepsies: From the benign to the disabling. Epilepsia Nov 2006;47 (Suppl 2):62-66). (Respond: Dr Giuseppe Gobbi, email: giuseppe.gobbi@ausl.bologna.it).

COMMENT. Recent studies have questioned the benign nature of BCECTS and related syndromes, and have stressed the associated neuropsychological and cognitive deficits. These include attention (ADHD), visual-motor coordination, dyspraxia, language and learning disabilities, mental impairment, and behavioral disorders, usually concomitant with activation of interictal EEG discharges. BCECTS occurs as $15 \%$ of childhood epilepsies, the most frequent epilepsy in school age children. The genetic predisposition is frequent with male predominance. Seizures and EEG discharges resolve before the age of puberty, but cognitive and behavioral deficits often persist. 